CDD: 149.2

\title{
MODELS, TRUTH AND REALISM: ASSESSING BAS VAN FRAASSEN'S VIEWS ON SCIENTIFIC REPRESENTATION ${ }^{1}$
}

\author{
MICHEL GHINS \\ Institut Supérieur de Philosophie \\ Université Catholique de Louvain \\ BELGIQUE
}

michel.ghins@uclouvain.be

\begin{abstract}
This paper is devoted to an analysis of some aspects of Bas van Fraassen's views on representation. While I agree with most of his claims, I disagree on the following three issues. Firstly, I contend that some isomorphism (or at least homomorphism) between the representor and what is represented is a universal necessary condition for the success of any representation, even in the case of misrepresentation. Secondly, I argue that the so-called "semantic" or "model-theoretic" construal of theories does not give proper due to the role played by true propositions in successful representing practices. Thirdly, I attempt to show that the force of van Fraassen's pragmatic - and antirealist - "dissolution" of the "loss of reality objection" loses its bite when we realize that our cognitive contact with real phenomena is achieved not by representing but by expressing true propositions about them.
\end{abstract}

Keywords: Representation. Realism. van Fraassen. Model. Truth. Predication.

\footnotetext{
${ }^{1}$ I would like to thank in the first place the organizers of the Conference "Science, Truth and Consistency" (Campinas 23-28 august 2009) - in particular professor Itala d'Ottaviano - who give me the opportunity to express my intellectual debt and my admiration to professor Newton da Costa through this paper. I also wish to thank Bao Van Lan, Patrick Assir Toty, Isabelle Drouet, Leonardo Rolla, Olivier Sartenaer and Arne Vangheluwe who participated to my seminars on scientific representation held at the Institut Supérieur de Philosophie during the academic year 2009-2010.
}

Manuscrito - Rev. Int. Fil., Campinas, v. 34, n. 1, p. 207-232, jan.-jun. 2011. 
In his recent - and magnificent - book on scientific representation (2008) Bas van Fraassen examines important but often neglected aspects of various kinds of representation in different areas of human practice - such as art, caricature and cartography - and shows their relevance for understanding how scientific representation works. Far from trying to elaborate a "theory of representation", namely, a set of necessary and sufficient conditions for what counts as representation in general, van Fraassen aims at bringing to light the family resemblances - and differences - of successful and informative representing practices. Such investigation not only provides a wealth of novel insights on how science proceeds but also involves a reassessment of the ancient - but still well and alive - debate on scientific realism. By in large, I agree with most of the claims made by van Fraassen, but I tend disagree on three main issues. Firstly, I contend that some isomorphism (or at least homomorphism) between the representor and what is represented is a universal necessary condition for the success of any representation, even in the case of misrepresentation. Secondly, I will argue that the so-called "semantic" or "model-theoretic" construal of theories does not give proper due to the role played by true propositions in successful representing practices. Thirdly, I will attempt to show that the force of van Fraassen's pragmatic - and antirealist - "dissolution" of the "loss of reality objection" loses its bite when we realize that our cognitive contact with real phenomena is achieved not by representing but by expressing true propositions about them.

\section{REPRESENTATION}

\subsection{Fundamental Definitions}

According to the so-called "semantic" - or better "model-theoretic" - view of scientific theories, a theory is primarily (but not only) a set of models capable of representing some portions of reality, whether observable or not. 
"Representation" is a technical term mathematically defined in settheory. Patrick Suppes (2002, chapter 3) defines representation as an isomorphism (or at least homomorphism ${ }^{2}$ ) between structures.

A structure $S$ is a couple that involves two partners: the first is a set of elements $D$ called the "domain" and the second is a set of relations $r_{i}$ on the elements of this domain. A structure is symbolized (not represented!) thus:

$$
S=<D, r_{1}, r_{2}, r_{3} \ldots>\text { or } S=<D, r_{i}>\quad(1 \leq i)
$$

Although in the literature "structure" is often employed to designate only the set of relations, I will use the term "structure" to refer to the couple made of the domain $D$ and the relations $r_{i}$. In order to refer specifically to the set of relations $r_{i}$, I will use the words "organization" or "form".

Two structures $S=<D, r_{i}>$ and $S^{\prime}=<D^{\prime}, r^{\prime}{ }_{i}>$ are, by definition, isomorphic just in case there exists a one-one function $f$ such that for all $r_{i}$ and for all n-uple $\left(a_{1}, \ldots, a_{n}\right)$ of elements in $D$ that stand in the relation $r_{i}$ there exists a n-uple $\left(a_{1}^{\prime}=f\left(a_{1}\right), \ldots, a_{n}^{\prime}=f\left(a_{n}\right)\right)$ of elements in $D$ ' that stand in the relation $r_{i}^{\prime}$. " $f$ " is called the "representative function". For a relation of representation between two structures to obtain, we must decide if $S$ represents $S^{\prime}$, or, on the contrary, if $S^{\prime}$ represents $S$. This asymmetry condition entails a distinction between a representing structure and a represented structure. Notice that in the case of isomorphic structures, they do not possess intrinsic characteristics that would give us reasons choose $S$ rather than $S^{\prime}$ as the representing structure (usually called the "representation"). The asymmetry must come from "outside" by stipulating that, say, $S$ represents $S^{\prime}$. Such an asymmetry is of course captured by specifying

\footnotetext{
${ }^{2} \mathrm{An}$ isomorphism is a one-one function, whereas an homomorphism is a many-one function. (Suppes (2002, p. 58.))
} 
that the domain of the representative function $f$ is $D$ and its set of values is $D^{\prime}$.

It is frequently observed that claiming that two structures are isomorphic without paying attention to the specific properties of their elements and the relations between them is just saying that they have the same cardinality as Max Newman proved in 1928. In order to overcome this difficulty Bertrand Russell introduced the distinction between abstract and concrete structures. A concrete structure is characterized by means of the specific properties of its elements and the specific relations in which these stand ${ }^{3}$. At the abstract level, the existence of an isomorphism between structures implies that their second-order properties are identical. But in the case of isomorphism between concrete structures we do not in general have identity of form since the specific relations taken into account in the respective domains may be different. The construction of a representative function between two concrete (as opposed to mathematical) structures always involves some abstraction: not all properties and relations are taken into account. If a representative (isomorphic or homomorphic ${ }^{4}$ ) function has been constructed between two structures, they are said to be "structurally similar" ${ }^{\text {. As }}$

\footnotetext{
${ }^{3}$ For a clear presentation of the distinction between abstract and concrete structures, one may consult Chakravartty (2007, p. 36-39).

${ }^{4} \mathrm{An}$ homomorphism is a many-one form preserving function.

${ }^{5}$ We may also consider structures for which the relations of interest are not defined on all elements of the targeted domain. In this case, one speaks of "partial structure". "The central idea is that in a partial structure, the relations and operations are defined for only some elements of the domain" (Da Costa French (2003, p. 19)). We may also be ignorant whether these relations hold or not between some elements. A careful presentation of the notions of "partial structure" and "partial isomorphism" can be found in Da costa and French (2003). These refinements are especially useful when tackling the question of the evolution of scientific theories and the heuristic role of models. The oversimplified presentation of structural similarity I offer here does not significantly affect the issues addressed in this paper. "Partial structure" should not be confused with "partial representation". A repre-
} 
an example, consider the concrete structure consisting in a set of five parallel straight lines on which lie oval black patches. The black patches and lines are elements of a domain $O$ that are organized by precise spatial relations $r_{1}, \ldots, r_{m}$. Call this structure $S=\left\langle O, r_{1}, \ldots, r_{m}\right\rangle$. A melody is a set of specific music notes $N$ organized in a temporal sequence. Call this structure $M=<N, r^{*}{ }_{1}, \ldots, r^{*}{ }_{m}>$. A music score is a succesful representation of a melody provided an isomorphism has been constructed between the two. This is a necessary but not sufficient condition, since other conditions have to be realized for the representation to be successful, as we will see below. Notice that notes with the same pitch are not taken to be identical since they occur at different moments of time ${ }^{6}$. It is a matter of practical decision to choose $S$ to represent $M$ or vice-versa. (We could ask if some actual performance is a correct representation of a music score).

It is time to introduce the notion of "model". A model is in the first place a structure that makes true or satisfies a set of statements. Thus, some structure of measurement numbers makes true the statement "Brazil's birth-rate is higher than Belgium's". On the other hand, scientists often stress the representative role of models. In this second sense, models are the possible representors of structures similar to them. Da Costa and French insist on what they aptly call this "dual role of models" (Da Costa and French (2003, p. 33)). Pursuing in this direction, we may construe scientific theories as classes of models that satisfy some statements (e. g. axioms if the theory is axiomatized) and are possible representations of some concrete structures.

sentation is partial or incomplete when some properties and relations of the representor and the represented are disregarded as the result of abstraction, which is always the case outside pure mathematics.

${ }^{6}$ The pitch of the corresponding note is determined by the spatial relation between the black patch and the lines; but I do not wish to enter into too many details... 


\subsection{Informative representation}

Models in science are supposed to convey some knowledge or information on concrete systems. When scientists construct models, their ambition is to correctly represent - partially at least - real concrete systems. A representation is always partial since only some aspects (properties and relations) of the system can be taken into account in the model. A representor - a model - is useful provided its user manages to gather from it some information about the represented in a certain context. In practice, the representational relation is a fourplace relation which involves the model, the represented, the user and the context. $S$ represents $S^{\prime}$ only for a user $U$ in a proper context $C$.

The first step in the construction of a successful representation is to construct a mapping between two entities (things, fields, processes etc.). To achieve this, we have to look at these entities as composed of some parts, that is, we have to consider them as sets of elements. If we want to construct a mapping which is also a representative function, we must focus on some relations between the elements of the respective entities. In other words, we must look at the entities as forming a structure or a system. A system is nothing else but a concrete structure. To see entities as systems is an inescapable prelude to any scientific investigation. Such an attitude with respect to entities in general is present at the very beginning of any scientific démarche and may be called the "original" or "inaugural" abstraction (Ghins (2009)). Looking at entities as systems is a precondition of what Bas van Fraassen calls the "objective attitude" (van Fraassen (2002)) ${ }^{7}$ which lies at the core of scientific practice.

\footnotetext{
${ }^{7}$ Looking at an entity as a system certainly does not imply that the entity is not really a system. Analogously, looking at a tree as having leaves (and disregarding many of its other properties) does not imply that it has no leaves.
}

Manuscrito - Rev. Int. Fil., Campinas, v. 34, n. 1, p. 207-232, jan.-jun. 2011. 
In order to be able to gather information from the representor, its user must further know how to map specific elements of the representor into the represented system. This presupposes that the user can identify the relevant elements both in the representor and the represented by relying on some of their individual properties such as being a black patch standing in specific spatial relations and being a specific music note.

In practice, we are thus interested in some selected properties of the elements of the respective domains and also in some selected relations purportedly holding between the elements in the two structures (model and represented) involved. Suppose one is interested, as a UN population expert for example, firstly, in countries, secondly, in their birth-rates, thirdly, in a partial ordering of these countries according to their birth-rates. As a precondition, the UN expert must be able to identify and distinguish entities called "countries" from other entities such as pineapples, saxophones etc. In order to achieve this, she has to rely on some properties possessed by countries, such as having a territory, a constitution etc. The UN expert also has reasons to believe that each country has a population and also a birth-rate which can be measured and expressed by a number (the number of births per year for one thousand inhabitants). The birth-rates can then be partially ordered by means of the relation " $\mathrm{x}$ is greater or equal than $\mathrm{y}$ ".

Suppose the UN expert delivers a Powerpoint presentation at a conference on world population. Typically, a slide will contain two columns: one with words and the other with numbers. We are told, or we know from our background knowledge, that the words are names of countries and that the numbers designate birth-rates. On the same row, we will have the name of a country and its corresponding birthrate. The numbers are spatially ranked from top to bottom: larger numbers are located above smaller numbers. 
For the speaker and her audience placed in such a context a slide functions as the representor of a structure of countries "in reality". Each row contains a name and a number that corresponds to a real country. The representative function here sends a row to a real country. The spatial organization of rows is supposed to reflect the ranking of countries according to their birth-rates. The representation is successful whether the assigned values for birth-rates are correct or not. A necessary condition for its success is that an isomorphic representative function has been constructed between the rows on the slide on the one hand and the countries birth-rates on the other hand.

It goes without saying that for the table to be informative the code must have been previously specified and made known to the audience. A code is a mapping between specific elements. Take the simple example of a ciphered message in which the letter "a" is always mapped into the letter "b", the letter "b" into the letter "c" etc. In the demographic example above, the rows of words and numbers are sent to birth-rates of countries. In other words, a concrete isomorphism has been constructed between the structure of the rows and the structure of birth-rates. By looking at the two columns slide and knowing the code, the persons in the audience can gather information which can be expressed in statements such as "Brazil's birth-rate is higher than Belgium's".

If we ignore the code, we may know from the context that a certain artefact has been used as a representation in a certain cultural environment, but we are unable to draw useful information from it. Take for example the following artefact "represented" in the picture below ${ }^{8}$ :

${ }^{8}$ This photograph is taken from Anthony J. P. Meyer (1995, p. 616, figure 709). The map is part of the collection of the Linden-Museum in Stuttgart. I am very grateful to Anthony Meyer, author of Oceanic Art (1995), and Dr. Ingrid Heermann, curator of the Oceanic art section of the Linden-Museum, for their kind authorization to reproduce this photograph. 


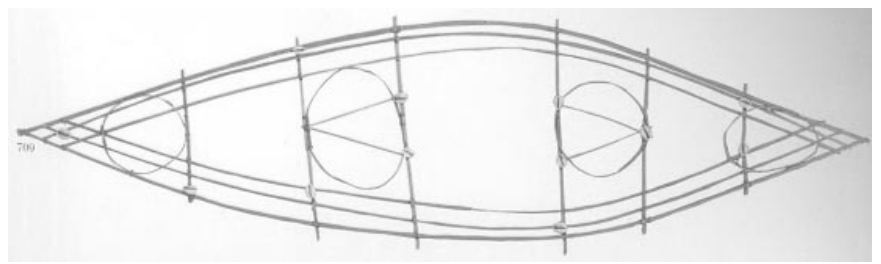

According to our background knowledge, we gather that this concrete structure has been used by the Micronesians of the Marshall Islands as a maritime map. We thus know that some elements of the map correspond to elements that are relevant for navigational purposes. The map is a system whose possible relevant elements are the wooden sticks, the cane circles, the shells, the knots etc. All these elements are identifiable by means of perceptual properties. We observe that the knots have different sizes and that the portions bounded by the knots or shells show various curvatures. But we ignore which properties were taken to be informative for navigation. What about the relations now? Are the relative lengths between two knots, the relative sizes of the knots etc. relevant or not?

Here are some factors that are surely important for sailing: the maritime currents and dominant winds, their location, extension, direction and strength. The relative positions of the islands - and perhaps their size too - can also be judged to be of interest. On the basis of our background knowledge, it is reasonable to suppose that the users of this handicraft established some sort of morphism between the map and the system of currents, winds and islands. But we are left in the dark as to what corresponds to what. Did they make shells or knots correspond to islands? May be; may be not. What about the relevant properties and relations? What was the representative function? Even we knew all that, we would still need crucial information, namely what 
Bas van Fraassen calls an "indexical" element ${ }^{9}$. Suppose I am about to sail from a beach on Ailinglaplap island. How do I locate myself on the map and how do I orient the map with respect to me and the surrounding landscape and stars?

This Micronesian example graphically teaches us that for this handicraft to function as a successful and also correct - and thus useful representation, plenty of information has to be imported from outside the map. For a concrete system to be practically used as a representation, some indications extrinsic to the system must be supplied. Some external indications are so obviously a matter of course for the natives immersed in a particular culture that they usually remain tacit or implicit. Their importance can easily be disregarded by the natives and are thus easily lost under significant cultural changes. In principle, such external information can be conveyed by means of statements in a language that the members of the community who use the representation understand. In itself, a system is never a representation. This is why any entity can be used to represent any other entity. As van Fraassen says in what he believes to be his main contention or Hauptsatz: "There is no representation except in the sense that some things are used, made or taken, to represent some things as thus or so." (2008, p. 23).

When we have established a representative function between a representor and a represented, we are entitled to speak of a similarity of structure between them. Similarity does not imply concrete identity. A spatial arrangement of coloured elements can represent a sonata. In picturing, which is a standard example of representing, the picture is a spatial arrangement of coloured elements and what it represents is also a set of spatially organized parts. In this case, we can speak of likeness or resemblance, since the elements of the two structures involved share

\footnotetext{
${ }^{9}$ This is why van Fraassen devotes considerable attention to perspective (see Ghins (2010)).
}

Manuscrito - Rev. Int. Fil., Campinas, v. 34, n. 1, p. 207-232, jan.-jun. 2011. 
relevant properties and also stand in the same kind (namely, spatial) relations. In my terminology, resemblance is a particular case of similarity. A representor resembles the represented when they share some specific first-order properties or relations of any kind (not necessary visual or geometrical $)^{10}$.

\subsection{Misrepresentation}

Among other examples, van Fraassen discusses Spott's caricature of Bismarck (2008, p.14) as a peacock. The drawing pictures some of Bismarck's facial features (he has a moustache, he is bald etc.) that allow the user to identify the referent of the caricature, namely Bismarck (and not Radowitz ${ }^{11}$ ). Spott's aim of course is that we do not see the drawing as a faithful portrait of Bismarck but as a caricature that represents him as vainglorious. How is Spott's aim achieved? van Fraassen rightly stresses that for the caricature to achieve its purpose some distorting of Bismarck's physical features is necessary. Bismarck's arms are replaced by wings, his chest looks like a long neck. He has a large spread out tail, which unavoidably makes us think of peacocks which in our culture are symbolically associated with vanity. The caricature is a misrepresentation in the sense that Bismarck is not a peacock. However, he is (perhaps) vainglorious.

Notice that the drawing misrepresents Bismarck only in the improbable event that it is taken to be a resembling portrait. On the other hand, the caricature does (ironically for sure) aim at representing a vainglorious Bismarck, just in the same way as a picture of a red apple can successfully represent a red apple, in the appropriate context. To put it shortly, the caricature is a representation of Bismarck on the basis of some physical resemblances with him but it represents him as

\footnotetext{
${ }^{10}$ van Fraassen uses the word "imaging" (2008, p. 34) (not necessarily visual) in order to refer to what I call resemblance.

${ }^{11}$ Radowitz was Bismarck's political enemy.
} 
vainglorious because the drawing of Bismarck includes features that resemble a peacock, which our cultural codes symbolically associate with vanity. Whether Bismarck was in fact vainglorious or not, the success of the representation relies on an established isomorphism between elements in the picture on the one hand and parts of Bismarck's body and features of peacocks on the other hand. Therefore, even in the case of misrepresentation, some isomorphism (or homormorphism) must be put in place by the user between the representor and the purported represented for the representation to be successful, although and on this I fully agree with van Fraassen - the presence of an isomorphism is far from being sufficient.

Thus I would contend that what is successfully represented by the drawing is not the "morphological" Bismarck (at least not mainly) but a vainglorious Bismarck. In our language, we may very well say, as van Fraassen does, that Bismarck is represented as vainglorious. What the "as" indicates in this context is that we disregard many of Bismarck's properties to focus on some of his physical and psychological properties. In other words, we abstract many properties to only select a few of them. Abstraction and selection which are key ingredients of any successful representation are like two faces of one and the same coin. Therefore, it is important not to confuse the Bismarck endowed with all his properties - the "whole Bismarck" - with one of the many possible "represented Bismarcks", at the plural. The referent of the caricature is the "whole Bismarck", whereas the "represented Bismarck" here is the "vainglorious Bismarck". One can speak of misrepresentation only when we compare Spott's drawing with another representor of the "physical Bismarck", such as a portrait, which would be taken to be more correct from a certain point of view. Misrepresentation has to do not with the adequacy of a given representor to the "whole Bismarck", but with a comparison of several representors according to certain purposes. If Spott's purpose were to offer a faithful portrait of 
Bismarck, that is a system of lines and patches corresponding to his morphological traits, then Spott's drawing would be a (partial) failure. But if his aim is to represent a vainglorious Bismarck, then Spott's drawing can be judged to be quite successful indeed.

\section{TRUTH}

Spott's caricature successfully represents a vainglorious Bismarck whether Bismarck was a real politician or a purely imagined character, and also irrespective of whether he was in fact vainglorious or not. Of course, the caricaturist wants the user to laugh at Bismarck. His aim is to ridicule him. The caricaturist presupposes that Bismarck was in fact vainglorious and such information can be gathered from the caricature in context and expressed in propositions. But, again, the caricature does successfully represent a vainglorious Bismarck, whether or not Bismarck was really vainglorious. Thus success does not imply the correctness of faithfulness of representation on this respect, even if the former is a pre-condition for the latter. However, both success and correctness rely on the supposed truth of some propositions.

Permit me to make a rather provocative claim: statements and propositions (which are the contents of statements) do not represent. Language does not represent the world! Such a contention appears objectionable - even offensive - only if are oblivious of the quite restrictive construal of representation I gave above. A necessary condition for an entity to function as a representor of another entity is that we establish a concrete morphism (isomorphism or homomorphism) between them. In this restrictive sense of representation, true propositions are not isomorphic representations of facts. In his Tractatus logico-philosophicus, Wittgenstein famously attempted to devise a theory of meaning as isomorphic representation. According to the "picture theory of meaning", a true elementary statement is a logical picture of a fact. The elements 
of an elementary statement, that is, the names, are mapped into elements, that is, corresponding objects (which are the name's referents) in a possible situation. The names stand in the same relations as the corresponding objects in the possible situation. If the statement is true, the objects actually stand in these relations and constitute a fact. According to this account of truth, statements and their corresponding facts share the same logical form. For Wittgenstein, the proposition is the statement in its projective relation to the world. From the statement (propositional sign), we can project a possible situation whose existence, a fact, would make the statement true. As we all know, the "picture theory of meaning" has been shown to be vulnerable to fatal objections, as Wittgenstein himself later acknowledged.

A major objection to the "picture theory of meaning" is that logical pictures or representors in general, do not possess any "illocutionary force": they do not assert or affirm. If I claim that an artefact represents a system of maritime currents in such and such a way, I assert something, and my assertion may be true or false. The point I want to stress is the following: I can use a concrete structure to successfully represent another concrete structure only if I make some (often implicit) assertions and if these assertions are true, irrespective of the correctness of the representation relatively to some specific purpose. For the sake of clarity, let us distinguish between two sets of propositions: first, the propositions that must be considered by the user to be true for the representation to be successful; second the propositions that must be true for the representation to be correct or faithful in some respect.

Turning to the example of countries and birth-rates, let us assume that the spatial ordering of rows correctly represents the ordering of the birth-rates of the countries in the world. For the representation to be successful some statements must be asserted and supposed to be true by convention. It is true - by convention - that names symbolize countries and that birth-rates are symbolized by numbers. Morevover, 
we implicitly assert that there are countries and that they do have a birth-rate. We also assume that it is possible to measure birth-rates and we assert that, for example, Brazil's birth-rate has value $b$. In fact, a row in the table can be interpreted in context as a predicative statement. This representation is correct - according to some criterion - if the propositions attributing birth-rate values to countries are (approximately) true. The model can be said to be faithful only if such statements are true. Their truth is a condition of possibility of the correctness of the representation, and not the other way around. Truth then is more fundamental than representation.

Of course, a model can be incorrect. For example a model which would assign to Belgium a higher birth-rate than Brazil's would be incorrect in this respect. Nevertheless, given the conventions in place, the model represents a possible concrete structure. It is successful in the sense that the representor can be appropriated by a user in a given context to represent a specific possible concrete system. On the basis of her successful appropriation, the user can gather some information about reality in the form of statements that she may accept as true or submit to further scrutiny. For example, she may decide to perform updated measurements of the birth-rates of some countries.

The point here is that for a representation to be correct or incorrect there must be representation in the first place. Correct representing in some respect presupposes successful representing. And success relies on the - at least supposed - truth of some statements. That there are rows with names and numbers in the representor, that there exist countries with some birth-rates and so forth. Given the identification of the representor, the represented and the representative function between them by a user in context, success is achieved. Then, and only then, the issue of correctness - in some respects - can be raised at all.

Consider again Spott's caricature of Bismarck. Suppose that a user, given her lack of information on their physical appearances, takes the 
target of the representation to be Radowitz. Is her representation successful? Yes, in a sense. It might even be correct (in this respect) if Radowitz is actually vainglorious. This success again relies on the truth of some propositions: the caricature contains elements that are like parts of a human and peacock's body, peacocks in our culture are associated with vanity etc. Radowitz is a man (and not a woman or a young boy), he is a politician (like Bismarck) and so on. Thus, even for someone who (wrongly) identifies the target of Spott's caricature, success in representation depends on the truth of some propositions.

Talking about pictures, van Fraassen says: "(...) it is hard to accept that a picture could fail to convey anything correct or true about something and still be a picture of that thing" $(2008,16)$. This holds true for any kind of representation. And since anything can be used to represent anything, success in representing must always rely on some truths that convey some information about its target. Therefore, a successful representation is always correct in some respects. If we ask about the correctness of a representation, this question is necessarily raised by a user in some context. Thus, the distinction stated above between propositions whose truth warrant success and propositions that provide correct information is highly contextual.

It is hardly disputable that the question of the faithfulness of a representation only arises relatively to some specific interest or purpose. With respect to Spott's caricature we may ask: was Bismarck really vainglorious? But we may also ask: did he really have such a big moustache? The same kind of questions can of course be asked about Radowitz. In science, we are interested in the correctness of a model relatively to some specific information. Is the representation of the birth-rates of countries correct? Well, we want to know whether a 
specific country actually has the indicated value for its birth-rate. In other words, can we draw some interesting truths from the representor?

I suspect that underestimating the role of true propositions in both the success and correctness or representation flows from embracing the "semantic" or "model-theoretic" view of scientific theories in too restrictive a sense. It surely would be unfair to the proponents of the semantic view to accuse them of neglecting the importance of (true) propositions for science. However, since models "occupy centre stage" (Fraassen 1980, 44) they force propositions to recede to the periphery, so to speak. Far from undervaluing the importance of models in scientific theories, I tend to believe that propositions are more fundamental, since the success and the correctness of our representations are grounded on true propositions, and not the converse.

\section{REALISM}

In science, in order to construct a model of a phenomenon, an observed triangle say, we must in the first place look at the triangle as a system. This is the "inaugural" abstraction mentioned above. Then, by performing a further abstracting move, we construct what I will call a "phenomenal structure", such as the set of the perceived lengths of the sides of the triangle organized by the relation " $\mathrm{x}$ is equal or larger than y". The next step is to measure the sides of the triangles and assign them numbers in some unit in order to construct a "data model" isomorphic to the phenomenal structure. But the scientist does not stop there. Typically, the data model is associated, again by means of an isomorphism, to what van Fraassen calls an "empirical structure". The empirical structure belongs to a wider mathematical structure, namely a theory, which in this case is the Euclidean vector space. The empirical structure is of course also theoretical, since it is a substructure of the Euclidean space which is a theoretical structure if anything is. If 
we have managed to construct an isomorphism between the empirical substructure and the data model ${ }^{12}$ in such a way that the measured values sufficiently fit the theoretical values, we have embedded the data model in the theory.

The overall picture can be schematized thus:

Phenomenon

$\downarrow$ (abstraction)

Phenomenal structure

$\approx($ isomorphism $)$

Data model

$\approx($ isomorphism $)$

Empirical substructure

$\subseteq($ inclusion $)$

Wider theoretical structure

\footnotetext{
${ }^{12}$ When the data are in finite number and are values for continous quantities (such as the pressure in function of the volume for a gas) they are "smoothed out" to obtain a "surface model" (van Fraassen 2008, p. 167) which can then be compared to the theoretical curve (e.g. Boyle-Mariotte's law).
} 
Within this general framework, the issue of scientific realism can be raised at three distinct levels. Traditionally, the philosophical debate has mainly focussed on the reality of the non-empirical superstructures which are non-observational but are supposed to explain, for example causally, the structure of data. At this level, philosophers argue over the existence of unobservable entities such as electrons and genes. At a second level, we may ask if the data structure fits the empirical substructure to a sufficient degree of accuracy. In other words: is the theory empirically adequate? The third level concerns the relation between the "phenomenal structure" and the phenomenon. Although this question is less frequently explored by philosophers of science, it certainly is the most fundamental.

To van Fraassen's credit, this third issue is addressed at length in chapter eleven of Scientific Representation and honoured with a special name, the "Loss of Reality Objection":

"How can an abstract entity, such as a mathematical structure, represent something that is not abstract, something in nature?" (2008, 240).

Unlike van Fraassen (2010, p. 547) I insist that scientific representation can only take place between structures. Strictly speaking, we never represent phenomena since these are immediately given in observation, not as systems or structures, but as totalities or 'wholes'. Yet, some of our representations do provide some useful information about phenomena. This information can be expressed by means of true propositions, such as: this side of the triangle is $10 \mathrm{~cm}$ long. Predicative propositions of this kind (a relation term is a many-place predicate) are not representations, neither do they assert that some representation is accurate. When we attribute a property (denoted by a predicate term) to a thing, we do not represent the thing as possessing a property. Whatever a predicative judgement is, it does not state a representative relationship between a property on the one hand and a thing on the 
other hand (and even less a relation between an "image" or "representation" in my mind and a thing). When I assert a proposition and make a predicative judgement there is no chasm between a representation and a phenomenon, simply because I do not represent, period. In a judgement we attribute some properties to phenomenal entities. The representational procedure starts from phenomenal entities and some abstracted properties truly (at least supposedly) possessed by them. Then - and only then - the representational activity can proceed with the construction of homomorphic structures such as phenomenal structures, data models and empirical substructures.

But what is the relation between a - representative - theory and the phenomenon then?

"For us the claims

(A) that the theory is adequate to the phenomenon and the claim

(B) that it is adequate to the phenomenon as represented, i.e. as represented by us,

are indeed the same!" $(2008,259)$.

van Fraassen here has in mind the adequacy of a theory to some phenomena, but the question can be raised for data models as well. Indeed - for us - it would be inconsistent to doubt the adequacy of a data model to the phenomenon when the phenomenal structure is adequately represented by the data model. We might of course be mistaken since other data gathered with the aid of more precise instruments can later put in doubt the accuracy of previous measurement results. This would lead to the construction of another representation which may be more accurate than the previous one. Such worries however are epistemological whereas the loss of reality objection is metaphysical. Do we loose contact with a real phenomenon when we construct representations of it? For van Fraassen such qualms are out of order. The possibility of meaningfully asking if a representation "really' corresponds to the "real" phenomenon hangs upon the purported possibility of bracketing 
the indexical aspect of our representation and putting ourselves in a "godlike point of view" or a "view from nowhere". To contemplate the reality as it is an sich in a sort of survol cartésien is indeed inaccessible to us. By resorting to pragmatics, van Fraassen says: "That $(A)$ and (B) are the same is a pragmatic tautology. (...) this removes the basis for the loss of reality objection." (2008, 259) Dissolving metaphysical puzzles by resorting to pragmatics is a classical empiricist manoeuvre. Yet, in the hands of van Fraassen such a ploy acquires considerable ingenuity and force.

If we believe that we are prisoners of our representations, then asserting (B) and denying (A) would indeed plunge us into the hot waters of pragmatic incoherence. This would be tantamount to claiming that my theory is empirically adequate but I don't believe that it is. However, the debate here does not revolve around the relation between structures, namely an empirical substructure on the one hand and a data model or a phenomenal structure on the other hand, but on the relation between the phenomenal structure and the phenomenon. This is why I'm puzzled when I read that some elements of theoretical models (the empirical substructures) "are meant to represent the observable phenomena" $(2008,289)$. This certainly is in line van Fraassen's previous writings: a theory primarily is a class of models some parts of which - its empirical substructures - are possibly homomorphic to data models (or, more accurately surface models). But this contention does not seem to square very well with van Fraassen's newly introduced distinction between phenomena and appearances: "Phenomena will be observable entities (objects, events, processes). Thus 'observable phenomenon' is redundant in my usage. Appearances will be the contents of observation or measurement outcomes." (2008, 8) Thus, appearances are structures (phenomenal structures or data models), whereas phenomena are not. 
"(...) the phenomena can be measured and observed in different ways" $(2008,289)$. Therefore, appearances are various perspectives on the same real phenomenon.

According to van Fraassen, a successful representation is always an action performed by a user. Therefore, since representation presupposes abstraction, abstraction always involves an indexical ingredient. I agree with this. We may say that abstraction is indexicality-laden. The phenomenal structure and other appearances are constructed and understood by a user in a certain context. Therefore, it seems misleading to call them abstract; they perhaps may be called abstracted structures, but they are not merely mathematical, they are concrete structures. Their concreteness springs from the fact that they are used in a certain context. A subway map for example is a mathematical (geometrical) structure, but it becomes a concrete structure when it is appropriated by a user who gives some meaning to the lines and round patches of the map, and also locates himself with respect to it.

We could then rephrase the claims (A) and (B) above thus:

$\left(\mathrm{A}^{\prime}\right)$ the map is adequate to the subway network

(B') the map is adequate to the subway network as represented, i.e. as represented by me.

Granted, I cannot assert (A') and deny (B'), and vice-versa. But the reason I cannot is that we are moving ourselves in a world of admittedly concrete - structures. I have established a representative function - albeit implicitly - between a map (the representor) and the subway (the represented). If correct, the map is useful and helps me to reach the destination I want. But, as we saw, the faithfulness of a representation rests on true propositions. Let's come back to the simple example of the triangle. Is it true that this object given in perception has three sides? Yes. Is it true that each side has a length? Yes. If so, I can construct a (concrete) phenomenal structure of lengths that are organized by means of a partial ordering. As a next step, I can 
also construct a (concrete) numerical structure (a data model) whose elements are lengths measured in some unit. The indexical element is of course always present, but it is not idiosyncratic. Many other observers can ascertain those basic facts about a perceived triangle. In the same way, a subway map can be efficiently appropriated by many different users.

I submit that propositions such as "this phenomenal entity has three sides" are true in the sense of correspondence. That is, there is something independent of us that renders those statements true. I cannot flatter myself to be a truth-maker of these propositions. Let's call truth-makers of propositions "facts". The exact nature of "independence", "fact", "correspondence" and so on need not bother us too much at this point. For sure - and this is crucial - the correspondence between a true proposition and a fact is not to be understood in terms of homomorphism and representation.

Is it true then that the phenomenal triangle is a system? Yes. In this sense, we can also claim that triangular systems exist. The fact that we have constructed an abstracted structure does not imply that there is no system in reality. To assert that a certain system exists here is tantamount to asserting that the sides of the triangle are in fact organized by a relation of spatial ordering. That's all. Such a contention does not imply that there are properties or relations existing in some sense independently of us nor that they "cut nature at its joints". The existence of properties and furthermore, the existence of natural properties or kinds, do not have a direct incidence on the existence of systems in the sense just explicated.

The upshot of the above discussion is the following. If we pay sufficient attention to the truth of the propositions that ground the success and correctness of our representing practices, the loss of reality objection is not dissolved, but solved. Reality is retrieved because actually it never was lost. Phenomena are not represented by abstract and not 
even concrete structures because phenomena are not systems or structures, and only structures can be represented. If it is also true that a phenomenal triangle has lengths, then some structures are models which convey useful information about this phenomenal thing, without representing it. Such a claim may sound paradoxical, even provocative, but so be it.

Consequently, a realist - or a metaphysician...- such as myself who believes that there are things out there in the world, does not "insinuate (...) that there is a relation between data model and phenomena, which determines whether the data model represents the phenomena, and which has nothing to do with anything but the two of them" (van Fraassen 2008, p. 252) simply because, for such a realist, there can't possibly be a representation of a phenomenon by a data model. Yet, useful data models can be constructed by means of true propositions about phenomena. Of course, we can continue to employ the word "represent" in the case of the construction of representations that convey in context - useful information about phenomena. But we must be wary that this use of representation is not based on some selected morphism between structures but, again, only on the truth of some propositions.

One thing that twentieth century philosophy taught us is to be wary of the traps laid by the language we use. Speaking of the representation of a thing is fatally misleading. What is represented is what we decide to abstract from the entity we refer to, not the entity itself. We loose touch with reality only if we remain prisoners of the world of representations and homomorphic putative relationships among these. (We can no longer be saved by a Cartesian god who warrants the faithfulness of our ideas or representations to some real systems). Talk of representation keeps us confined to the realm of phenomenal structures, appearances, data models, surface models, empirical structures 
and so on. If we recall that our representative practices are based on true propositions, we realize that our contact with reality has never been severed.

\section{CONCLUSION}

In the course of these austere reflections, I have been trying to show that our successful representations always rest on some morphism that we establish between concrete structures. Furthermore, the success and correctness of our representations rely on the truth of some propositions. As a consequence, truth seems more basic than representation. If we take a representation to convey some useful information, we must presuppose that some propositions are true. This applies in the first place to the level of observable phenomena. The construction of a representation that has some phenomenon as its referent capitalizes on some true propositions about this referent. Although we are unable stricto sensu to represent phenomena, we do manage to construct representations that convey useful information provided we have made some true judgements about those phenomena. Our observable access to real phenomena is the soil of our true judgements about them. It is only on such ground that we are in touch with reality and that we can develop successful scientific representing practices. Issues such as the fitting of theory to data model and the purported existence of non-observable entities come next. We can tackle these questions meaningfully only if we have managed to reach some truths about phenomena and achieve success at the level of our representation of phenomenal structures. 


\section{REFERENCES}

CHAKRAVARTTY, A. A Metaphysics for Scientific Realism. Knowing the Unobservable. Cambridge: Cambridge University Press, 2007.

DA COSTA, N. FRENCH, S. Science and Partial Truth. A Unitary Approach to Models and Scientific Reasoning. Oxford: Oxford University Press, 2003.

GHINS, M. "Realism", online Interdisciplinary Encyclopaedia of Religion and Science. http://www.inters.org, 2009.

- "Bas van Fraassen on Scientific Representation", Analysis, 70, p. 524-536, 2010.

NEWMAN, M. H. A. "Mr. Russell's 'Causal Theory of Perception' ", Mind, 37: p. 137-48, 1928.

MEYER, A. J. P. Oceanic Art - Ozeanische Kunst-Art océanien. Cologne: Könemann, 1995.

SUPPES, P. Representation and Invariance of Scientific Structures. Stanford: CLSI, 2002.

VAN FRAASSEN, B. The Scientific Image. Oxford: Oxford University Press, 1980.

. The Empirical Stance. New Haven: Yale University Press, 2002 .

. Scientific representation. Paradoxes of Perspective. Oxford: Oxford University Press, 2008.

- "Reply to Contessa, Ghins and Healey", Analysis, 70, p. 547556,2010 . 